\title{
End-Stop Exemplar Based Recognition
}

\author{
Søren I. Olsen \\ Department of Computer Science, University of Copenhagen, Denmark
}

\begin{abstract}
An approach to exemplar based recognition of visual shapes is presented. The shape information is described by attributed interest points (keys) detected by an end-stop operator. The attributes describe the statistics of lines and edges local to the interest point, the position of neighboring interest points, and (in the training phase) a list of recognition names. Recognition is made by a simple voting procedure. Preliminary experiments indicate that the recognition is robust to noise, small deformations, background clutter and partial occlusion.
\end{abstract}

\section{Introduction}

Recognition of objects in images has been a central topic in computer vision for decades. The space here does not allow a review of the many important contributions. A few influential papers for the present work is [1-8]. The problem addressed is to name objects in an image according to the shape information in the image based on previously learned images. It is not here a goal to localize the instance(s) of a recognized shape. Some of the problems that need to be addressed are: 1) How may shape information be represented, how is this information detected, and how stable is the representation to different kind of distortions? 2 )How can the relation between image shape information and the (possibly ambiguous) naming's be learned such that the discriminability is high and generalization possible? 3) How can the shape information be matched computationally efficiently to previously registered information?

Previous work [5] on models for End-stopped cells (here named End-stops or ES) in the visual cortex has been a strong inspiration for the present work. Endstops mark the positions in an image where one or several structures (edges or lines) end, e.g. corners, junctions etc. End-stops have several pleasing features: They are fairly robust to noise and they may be attributed with the direction of the structures pointing from the ES. Thus, they carry rich information on the local image shape. By using interest points attributed with local shape information, the present work falls in the same category as $[2,6,8]$ and differs from the so-called appearance-based methods. Also, relations exist to the method [7] where (non-local) edge structures are used as the key elements. One advantage of using locally defined keys is that recognition of partially occluded objects is eased. Also the variation in shape deformation local to the interest point can be assumed limited. One disadvantage is that the discriminative power is reduced [3]. A solution to this problem is to attribute each End-stop with more global information, e.g. the position of neighboring interest points. Although 
an occurrence of a specific ES may be frequent, the occurrence of two or more specific ES in a specific configuration is rare [1]. Another technique is to apply a scale-space-approach. Here the image is analyzed at different resolutions and the information from larger scales used to supplement the local information [6]. The present approach use both techniques.

A number of methods have used interest points attributed with features invariant to a class of geometric transformations [8]. This may reduce the number of images needed to be represented if an $3 \mathrm{D}$ object is to be recognized from a large span of views, but requires that the measures can be obtained accurately. The present approach follows this line only to a limited extend. Although some attributes are chosen insensitive to small deformations, no attempt is made to facilitate matching wrt. a large class of transformations. Instead, the recognition is limited to previously seen similar exemplars. The disadvantage is twofold: First, the database of learned shapes must be larger. Thus, the learning step becomes more demanding. Secondly, the search space for the matching is enlarged. To limit the computational burden this space must be pruned efficiently but carefully. In the present approach a sequence of gradually more selective and computationally more demanding matching steps are performed. Similarly, the attributes used are selected gradually more expressive and less local.

When entering (learning) new images into the system a list of name-tokens, corresponding to the possible interpretations are attributed each ES. All ES in the image are attributed with references to the listed names, because we cannot know in advance what names to link to what structures. In the present work no attempt is made to learn which ES are important for a recognition and which ES belongs to irrelevant details or background clutter. However, this will be an obvious extension in future work.

\section{Feature point detection}

The first step in detecting the End-stops in an image is to measure the orientation energy for each pixel and for each orientation (8 orientations are used). This is computed by the 2-norm of the response to an edge- and a ridge-filter chosen as scaled oriented first and second derivatives of a Gaussian. Then a non-maximum suppression is applied. Thus each pixel is classified as showing no structure or a structure with an orientation and an energy. Then, for each orientation 2 new convolutions (differing in $180^{\circ}$ corresponding to 2 out of 16 directions) are made on the orientation energy. Informally, the filter used here is 'one-sided', narrow in orientation and long-tailed. The purpose of the filtering is to 'count' the number of structure-points located close to the specific direction and with an orientation similar to the direction [5]. This results (for each pixel) in a vector $F$, indexed by direction, of measures which have a high value if the image point is located on a locally straight structure with this orientation. In more detail let $\mathrm{P}$ be the image point under consideration and $S$ a neighboring structure point. Let $(u, d)$ be the polar coordinates of the vector $P S$ and let $v$ be the angle between the vector $P S$ and the structure orientation vector at $S$. Then the contribution from $\mathrm{S}$ is given 
by $\exp \left(-\frac{d^{2}}{2 \sigma_{d}^{2}}\right) \times \exp \left(-\frac{v^{2}}{2 \sigma_{v}^{2}}\right)$. By using linear interpolation this value contributes to $F[i]$ and $F[i+1]$ corresponding to the quantized directions $w_{i}$ and $w_{i+1}$ closest to $u$. From $F$ the end-stop-ness is computed by summing over all directions

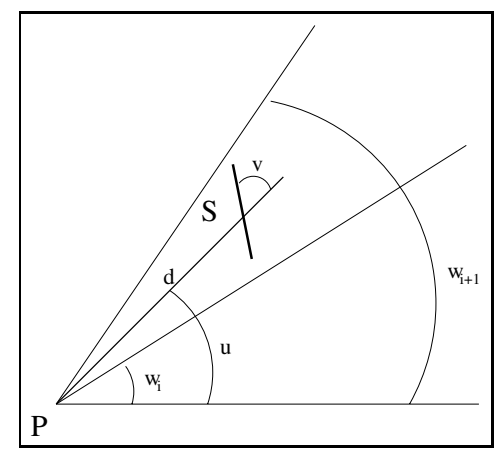

Fig. 1. Angles used in the computation of the contributions to the vector $F$. See text for further explanation.

the value for that direction minus the value for the opposite directions (if this difference is positive), i.e. as $\sum_{i} \max \{0, F[i]-(F[i+\pi]+F[i+\pi+1]+F[i+\pi-1])\}$ Thus, if an image point is located in the middle of an isolated straight structure the end-stop-ness will be zero. If located on the end of a straight structure the end-stop-ness will be high. If several structures end at the image point (corners or junctions) the end-stop-ness will be even higher. Finally, the End-stops are detected as the image points with locally maximal end-stop-ness. Figure 2 shows examples of detected ES in two images. End-stops mark asymmetrical structural occurrences. In addition points of maximal circularity are detected by a spatial non-maximum suppression of a measure $M$. First the sum $S_{d}$ over directions of contributions from structures oriented orthogonally to the direction and within a radial band specified by $d$ is computed for each pixel. Then $M$ is computed by maximizing $S_{d} / d$ over $d$.

By analyzing $F$ the direction of the ending structures (the legs of the ES) are found by the local maxima. Points of maximal circularity have no legs. Each ES is attributed a measure of asymmetry. First, for each direction $i$ the sum $D_{i}$ of distances to neighboring structure-points is computed. Then the asymmetry measure is computed as $\frac{1}{2} \sum\left|D_{i}-D_{i+\pi}\right| / \sum D_{i}$. This measure is zero if the local structure-points are positioned symmetrically and approaches 1.0 with the degree of asymmetry in positioning. The measure is insensitive to rotation and scaling. Also, each ES is attributed a 2-dimensional histogram representing the number of structure points with a specific orientation located at a specific direction from the ES. This attribute efficiently represents the structural and textural information local to the interest point. A similar representation has previously been reported in [4]. The attribute is insensitive to minor scale changes. Apart from orientation quantization problems matching of histograms for rotated ES is easy. Finally each ES is attributed with the polar coordinates of neighboring 



Fig. 2. Left: ES detected in a silhouette image of a slide gauge; Right: Detected ES in an image of a painting

ES. This information is necessary to differentiate between locally similar but globally different shapes. To be registered in the database, an ES is required to have at least 3 neighbors.

Due to noise and irrelevant details some structures are not detectable at high scale resolution. To solve this problem and to make possible recognition of objects scaled significantly in size End-stops are detected at a number of scale levels computed by successive convolutions with a Gaussian and by sub-sampling. The sampling in scale-space has been chosen fairly dense (using a reduction factor of $1.25)$. The number of scale levels is determined from the image size. Typically $2-5$ levels are used. Inclusion of ES undetected at the present level but detected at coarser scale-levels in the list of neighboring ES, both supplement the necessary global information and makes the representation more robust.

\section{Shape recognition}

In the recognition phase each input ES is first matched to the ES-representations registered in the database. The matching is made in several steps. First the search space is limited by indexing a $n$-dimensional array, where $n \in[0: 3]$ is the number of ES legs, with the quantized directions of the legs. Bins corresponding to a rotation of \pm one quantization value (i.e. $22.5^{\circ}$ ) as well as bins corresponding to one or several quantization errors, or one missing leg, are checked as well. Experiments show that this may reduce the number of further comparisons by a factor of 10-20 without noticeably reducing the hit-rate. Next each input ES is compared to the stored representation in the union of checked bins. A fast and liberal matching based on the measure of asymmetry is used. By allowing a deviation of $50 \%$ the probability of missing the correct match is negligible while offering a further reduction of the search space by a factor of 5-10. The elements in the database that passes the test is compared to the input ES by a more extensive test and attributed a match score. The comparison is based on a 
$\chi^{2}$-test on the 2D-histogram of local structure point orientation located at some direction from the ES (similarly to [4]). The $\chi^{2}$-test is robustified by excluding histogram differences if these are based on very small numbers. Thus, spurious structure detections will not influence the test value. A match is accepted if the test value is below an empirically determined threshold. This is then converted to a match score $\in[0: 1]$ with 1 corresponding to a perfect match.

To further differentiate between the candidate ES matches the number of neighboring ES consistent with a neighbor to the candidate database ES is counted. First all pairs of potential neighbors are registered if they differ in relative orientation less than $30^{\circ}$ and in distance less than a factor of 1.25. Each candidate neighbor-match is attributed with its difference $\Delta O$ in orientation and scale ratio $\Delta d$. The first accepted neighbor-match is chosen as the one with the highest count of close neighbors in $(\Delta O, \Delta d)$-space (using a kernel based approach). Then the set of accepted neighbor matches is iteratively increased. In each iteration the current average of $(\Delta O, \Delta d)$ for accepted neighbor pairs is computed and the unmatched candidate pair closest to the current average is found. This is included if the variances of $\Delta O$ and $\Delta d$ for the extended set does not exceed empirically determined thresholds. Also, unmatched candidate neighbor pairs involving an ES in the newly accepted pair are deleted from the set of unmatched neighbor pairs. To allow for partial occlusion candidate ESmatches are not discarded if the number of matching neighbors is low. Instead, the match score is increased less than for candidate matches with a large number of matching neighbors. The net result is that each input ES is linked to zero, one or a few database ES, and that each link is attributed a match-score.

Then a simple voting procedure is applied. Each match votes with its score as strength, and has as many votes as there are names in the list of names associated with the database ES. The list of names are sorted and the top-ranking names selected. In the present study only the highest ranking name is considered. In a learning situation however, the user has the ability to select the correct name facilitating the system to learn what structures that are important for specific names, and what structures that are spurious and might be pruned.

\section{Experiments}

In the experiments reported below the system is first fed with a number of images, each annotated with one name-token, only allowing it to add new information. Then the system is tested by presenting it to new (or previously seen) unnamed images and the results of the classification is monitored.

In the first experiment 15 silhouette images of different tools were captured, and downscaled to a size of 256 by 256 . By using silhouette images the test focuses on recognition of geometrical shapes with few ES. Each image was analyzed, and the detected attributed End-stops entered into the database. A total of $181 \mathrm{ES}$ were installed. Then 4 of the images was selected and distorted with varying degree of rotation (up to $\pm 25^{\circ}$ ), of scaling (with a factor $\in[0.6,1.6]$ ), of skewing (up to $\pm 12^{\circ}$ ) and additive white Gaussian noise $(\sigma \leq 20)$. Also, tests 
were made using all types of distortion simultaneously. A total of 148 query images were generated. Of these 124 were recognized correctly. None were recognized as a wrong shape. Of the 24 unclassified images 13 were derived from an original image having few (9) ES. 11 of the unclassified images were deformed using a combination of all the distortion types. Also 29 new images, taken from a different viewpoint (view angle differing by $\approx 30^{\circ}$ ), of 6 of the tools (about half differently articulated) were captured, preprocessed similarly to the database images, and matched to the base. 24 of the new images were correctly recognized and 5 were left unrecognized. A general trend was that simple shapes (with few ES) were more sensitive to deformations, and that the recognition rate decreased with the amount of deformation. The number of false positives was constantly low (zero). To a large extent this may be due to the small size of the database. The results indicate that the system is fairly robust to small deformations, noise, and viewpoint changes.

In the second experiment 6 tool-images were randomly embedded 3 times in 5 different binarized texture images ( 450 by 450 pixels) resulting in 90 query images. When matched to the same database as in the first experiment $70 \mathrm{im}$ ages were named correctly. In 8 cases the correct naming was second in the name priority list, and in 1 case the image was left unclassified. 7 misclassification originated from the same tool-image, and 9 from the same texture image. On average about 1400 ES were detected in each query image. Due to the background clutter, a fraction of the ES originating from the tool-shape were distorted beyond recognition. Thus, the ratio of truly matching (undistorted) tool-ES to the total query-ES was low $(\approx 1 \%)$. To further test the robustness 3 tool-images were randomly embedded 2 times in 3 different textures such that only the left part of the tool-shapes were visible. The fraction of visibility was varied from 0.3 to 1.0 in steps of 0.1 . Of the 18 tests for each value of the visibility fraction $14,11,14$, $11,12,14,16$, and 17 images were correctly classified. Figure 3 shows examples of two successfully reconized shapes. In total 35 images of the 144 were incorrectly named. Of these 26 originated from the same tool image. Because the success rate depend both on the original image, the texture type, the actual embedding and on the selection of the visible part, the statistics are far from satisfactory to validate the robustness of the method. However, the results indicate that the system to some extent is robust to background clutter and partial visibility.

In the third experiment the tool-database was extended by adding 12 silhouette images of different toy animals, and 65 grey-scale images of different in- and outdoor scenes. Insertion of the 92 differently named images resulted in a database with 8847 registered ES. Then the originals were matched to the base. Of the 92 queries 91 were classified correctly. In one case the correct name was second in the list. This was the only case with several interpretations. This result indicates that the discriminative power of the representation is sufficient to allow a good classification for a somewhat larger database.

In the fourth experiment the car-database kindly provided by Prof. Shivani Agarwal, University of Illinois at Urbana-Champaign was used [2]. First the system was trained on 546 images showing one or several cars and 393 images showing no cars. The training images had a size of $100 \times 40$ pixels, and the 

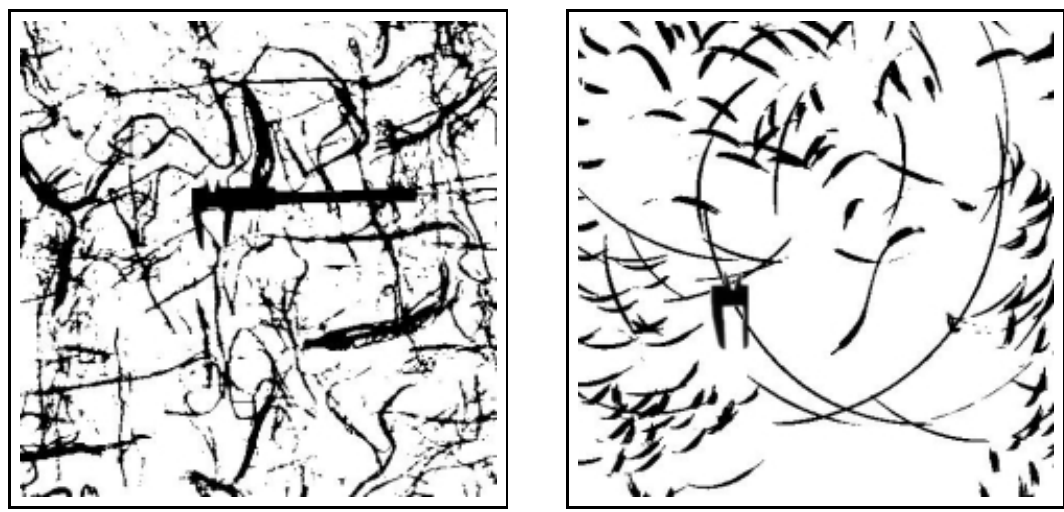

Fig. 3. Left: Shape embedded in texture; Right: Partial visible shape in texture. In both cases the shape was correctly recognized

resulting database contained $6420 \mathrm{ES}$. Then the system was tested on 170 new images all showing one or several cars and on 79 images showing no cars. The amount of clutter and occlusion for the positive query images was significant. In total there was 3 false negative and 19 false positives. A correctly classified image and the ES contributing to the classification is shown in Figure 4. In addition to the classification the program measured the confidence $C$ of the naming. This measure is based on the total support score $S_{0}$ for the most likely naming and computed by: $C=\left(S_{0} / \sum S_{i}\right) \times\left(1-\exp \left(\frac{s_{0}^{2}}{2 \sigma^{2}}\right)\right)$. The table below shows for sample point of $C$ the number $N_{t p}$ and percentages $P_{t p}$ of correctly classified cars with confidence value exceeding $C$, and the number $N_{f p}$ and percentages $P_{f p}$ of falsely classified cars with confidence value exceeding $C$.

Table 1. Reciever Operator Characteristics for the queries to the car-database.

\begin{tabular}{|l||r|r|r|r|r|r|r|}
\hline$C$ & 0.95 & 0.85 & 0.70 & 0.60 & 0.50 & 0.25 & 0.10 \\
\hline \hline$N_{t p}$ & 98 & 124 & 144 & 151 & 153 & 163 & 167 \\
\hline$P_{t p}$ & 57.6 & 72.9 & 84.7 & 88.8 & 90.0 & 95.9 & 98.2 \\
\hline \hline$N_{f p}$ & 1 & 1 & 2 & 3 & 4 & 7 & 13 \\
\hline$P_{f p}$ & 1.3 & 1.3 & 2.5 & 3.8 & 5.1 & 8.9 & 16.5 \\
\hline
\end{tabular}

The results show that the learned representation is sufficiently general to allow recognition of new shapes different but similar to previously learned shapes. Also, the number of false positives is small.

The program was implemented in $\mathrm{C}$, compiled using the gnu-compiler with -O option, and run on a $1.6 \mathrm{GHz}$ P4. No serious attempts were made to optimize the code. For an 256 (512) square image the feature extraction took 2-5 (10-30) seconds depending on the amount of image structure. The time for matching the set of extracted ES-descriptions to the ones in the database clearly depend on the size of both sets. For the larger database used in the third experiment 

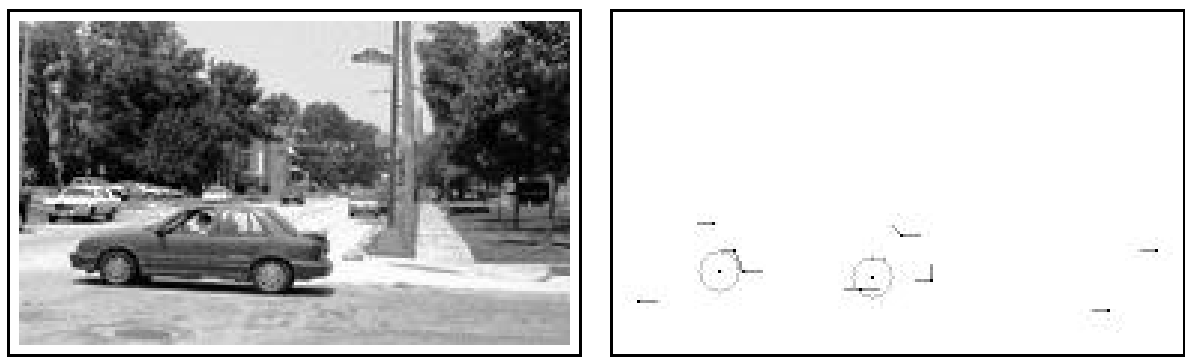

Fig. 4. A corretly classified test-image and the 11 features (7 true and 4 false) contributing to the classification.

(with $8847 \mathrm{ES}$ ) the matching time were roughly linear in the number of input ES, having an average of about $9 \mathrm{~ms}$ for matching one input ES. Surely these numbers can be lowered by optimizing the code.

\section{Conclusion}

An exemplar based recognition scheme using attributed End-stops as features has been described and a few preliminary experiments reported. The results indicate that the system is fairly robust to noise, small deformations, background clutter, and partial visibility. However, more experiments are needed to show the stability for a larger database of shapes. Also, the capability of the system to learn important structures from irrelevant details and background clutter (not dealt with in this paper) awaits examination.

\section{References}

1. Y. Amit, D. Geman: A Computational Model for Visual Selection, Neural Computation 11, 1999, pp. 1691-1715

2. S. Agerwal, D. Roth: Learning a Sparse Representation for Object Detection, Proc. 7 'th ECCV, 2002, LNGS 2353, pp. 113-127

3. A. Garg, S. Agerwal, T.S. Huang: Fusion of Global and Local Information for Object Detection, Proc. 6'th Int. Conf. on Pattern Recognition, vol. 2, 2002, pp. 723-726

4. S. Belongie, J. Malik, J. Puzicha: Shape Matching and Object Recognition Using Shape Contexts, IEEE trans. PAMI, (24)24, 2002, pp. 509-521

5. F. Heitger, L. Rosenthaler, R. Von der Heydt, E. Peterhans, O. Kubler: Simulation of Neural Contour Mechanisms: from Simple to End-stopped Cells, Vision Research vol. 32 , no. 5 , 1992 , pp. $963-981$

6. D. Lowe: Object Recognition from Local Scale-Invariant Features, Proc. 7 'th ICCV, 1999, pp. 1150-1157

7. R.C. Nelson, A. Selinger: Large-scale tests of a keyed, appearance-based 3-D object recognition system, Vision Research 38, 1998, pp. 2469-2488

8. C. Schmid, R. Mohr: Local Grayvalue Invariants for Image Retrieval, IEEE trans. PAMI, 19(5), 1997, pp.530-535 